\title{
Social isolation alters GABAA receptors plasticity and function: effect of voluntary consumption of ethanol Mariangela Serra* and Giovanni Biggio
}

Address: Department of Experimental Biology, Center of Excellence for Neurobiology of Drug Dependence, University of Cagliari, Cagliari, Italy * Corresponding author

from International Society on Brain and Behaviour: 3rd International Congress on Brain and Behaviour Thessaloniki, Greece. 28 November - 2 December 2007

Published: 17 April 2008

Annals of General Psychiatry 2008, 7(Suppl I):S25 doi:I0.II86/I744-859X-7-SI-S25

This abstract is available from: http://www.annals-general-psychiatry.com/content/7/SI/S25

(C) 2008 Serra and Biggio; licensee BioMed Central Ltd.

In social isolated rats brain and plasma levels of progesterone metabolites, $3 \alpha, 5 \alpha-$ TH PROG(AP) and $3 \alpha, 5 \alpha-$ THDOC (THDOC) are reduced while the positive effects of acute stress or acute ethanol on the content of these steroids are enhanced. The ethanol-induced increase in the abundance of AP is more pronounced in the brain than in the plasma of socially isolated rats. Accordingly, social isolation enhanced the effects of ethanol on the amounts of steroidogenic acute regulatory protein (StAR) mRNA and protein in the brain. Moreover, the ability of ethanol to inhibit isoniazid-induced convulsions is greater in socially isolated rats than in group- housed animals, an effect abolished by finasteride, an inhibitor of AP synthesis. In socially isolated rats, the amounts of the $\alpha 4$ and $\delta$ subunits of the GABAA receptor in the hippocampus were increased, an effect associated to an increase in GABAA receptor-mediated tonic inhibitory currents in granule cells of the dentate gyrus. Ethanol also increased the amplitude of GABAA receptor-mediated miniature inhibitory postsynaptic currents (mIPSCs) in CA1 pyramidal neurons with a greater potency in slices from socially isolated rats than in those from group-housed, an effect inhibited by finasteride.

According with the evidences that separation of rats from their peers during adolescence and adulthood increases voluntary ethanol consumption, isolated rats who had access to increasing concentrations of ethanol for 4 weeks, exhibited higher level of ethanol ingestion than grouphoused animals. Voluntary ethanol consumption during social isolation abolished the reduction of the brain and plasma content of AP and THDOC, the enhanced potency of ethanol on mIPSC recorded from CA1 pyramidal neurons, and the acute stress-induced increased in neuroactive steroid concentrations in the cerebral cortex and plasma.

These data suggest that the natural preference for ethanol may be related to the effect of this drug on the levels of neuroactive steroids, and that chronic stress due to social isolation may induce plastic adaptation of neuronal systems that contributes to a vulnerability to alcohol abuse. 